\title{
Short- and long-term health consequences of sleep disruption
}

\author{
Goran Medic ${ }^{1,2}$ \\ Micheline Wille' \\ Michiel EH Hemels' \\ 'Market Access, Horizon \\ Pharma B.V., Utrecht, ${ }^{2}$ Unit \\ of Pharmacoepidemiology \& \\ Pharmacoeconomics, Department of \\ Pharmacy, University of Groningen, \\ Groningen, The Netherlands
}

This article was published in the following Dove Press journal:

Nature and Science of Sleep

19 May 2017

Number of times this article has been viewed
Correspondence: Michiel EH Hemels Market Access, Horizon Pharma B.V., Graadt van Roggenweg 340, Building D, Fifth Floor, 353I AH Utrecht, The

Netherlands

Tel +3I 62970 04 II

Email mhemels@horizonpharma.com
Abstract: Sleep plays a vital role in brain function and systemic physiology across many body systems. Problems with sleep are widely prevalent and include deficits in quantity and quality of sleep; sleep problems that impact the continuity of sleep are collectively referred to as sleep disruptions. Numerous factors contribute to sleep disruption, ranging from lifestyle and environmental factors to sleep disorders and other medical conditions. Sleep disruptions have substantial adverse short- and long-term health consequences. A literature search was conducted to provide a nonsystematic review of these health consequences (this review was designed to be nonsystematic to better focus on the topics of interest due to the myriad parameters affected by sleep). Sleep disruption is associated with increased activity of the sympathetic nervous system and hypothalamic-pituitary-adrenal axis, metabolic effects, changes in circadian rhythms, and proinflammatory responses. In otherwise healthy adults, short-term consequences of sleep disruption include increased stress responsivity, somatic pain, reduced quality of life, emotional distress and mood disorders, and cognitive, memory, and performance deficits. For adolescents, psychosocial health, school performance, and risk-taking behaviors are impacted by sleep disruption. Behavioral problems and cognitive functioning are associated with sleep disruption in children. Long-term consequences of sleep disruption in otherwise healthy individuals include hypertension, dyslipidemia, cardiovascular disease, weight-related issues, metabolic syndrome, type 2 diabetes mellitus, and colorectal cancer. All-cause mortality is also increased in men with sleep disturbances. For those with underlying medical conditions, sleep disruption may diminish the health-related quality of life of children and adolescents and may worsen the severity of common gastrointestinal disorders. As a result of the potential consequences of sleep disruption, health care professionals should be cognizant of how managing underlying medical conditions may help to optimize sleep continuity and consider prescribing interventions that minimize sleep disruption.

Keywords: sleep, sleep disorders, children, adolescents, adults, health status

\section{Introduction}

Sleep is a biologic process that is essential for life and optimal health. Sleep plays a critical role in brain function and systemic physiology, including metabolism, appetite regulation, and the functioning of immune, hormonal, and cardiovascular systems. ${ }^{1,2}$ Normal healthy sleep is characterized by sufficient duration, good quality, appropriate timing and regularity, and the absence of sleep disturbances and disorders. ${ }^{3}$ Despite the importance of sleep, up to 70 million people in the US and 45 million people in Europe have a chronic sleep disorder that impacts daily functioning and health. ${ }^{2,4}$ For example, $\sim 20 \%$ of the serious injuries that result from car accidents can be associated with driver sleepiness, independent of the effects of alcohol. ${ }^{2}$ Lifestyle and environmental factors,

Nature and Science of Sleep 2017:9 |5|-|6| 
psychosocial issues, and medical conditions all contribute to sleep problems. ${ }^{2}$ There are $\sim 100$ sleep disorder classifications; however, they are typically manifested in one of the following three ways: failure to obtain the necessary amount or quality of sleep (sleep deprivation), an inability to maintain sleep continuity (disrupted sleep, also called sleep fragmentation, difficulty maintaining sleep, and middle insomnia), and events that occur during sleep (eg, sleep apnea, restless legs syndrome). ${ }^{2}$ The effects of sleep disorders on the body are numerous and widely varied across multiple body systems. This review focuses on the clinical consequences, both short term and long term, that result from disrupted sleep (not including short sleep duration) in adults, adolescents, and children who are otherwise healthy and in those who have an underlying medical condition. Information on basic science and mechanisms of these effects are included to provide background for the clinical outcomes, but are not thoroughly reviewed. Several recent reviews provide detailed information on the science and mechanisms of sleep disruption. ${ }^{5-7}$

\section{Methodology}

In order to better focus on the topics of interest among the myriad parameters affected by sleep, this review of the literature was designed to be nonsystematic. A search of Englishlanguage publications in the PubMed database was conducted in March and April 2016. Search terms were "caregiver AND sleep", "caregiver AND drug administration", "insomnia", "middle insomnia", "restless leg[s] syndrome", "sleep AND drug administration”, "sleep apnea”, "sleep continuity”, “sleep deprivation”, “sleep disorder”, "sleep disruption”, "sleep disturbance", "sleep fragmentation", and "sleep maintenance". Together, these search terms generated over 60,000 hits. For each individual search, we reviewed the most recent articles to identify those that specifically discussed the consequences of disrupted sleep, rather than those of short sleep duration or other sleep problems. For topics that were not adequately covered by recent literature (previous $\sim 5-10$ years), we looked slightly further back in the literature. Other publications were identified by examining the reference lists of publications included in the literature searches. The websites of the American Academy of Sleep Medicine, Sleep Research Society, and the European Sleep Research Society were also searched for additional publications. This nonsystematic review pulled information from a total of 97 references.

\section{Characteristics of normal sleep}

The stages of sleep have historically been divided into one stage of rapid eye movement (REM) sleep and four stages
(Stages 1-4) of non-rapid eye movement (NREM) sleep that are characterized by increasing sleep depth. ${ }^{2,8}$ The deeper sleep stages (Stages 3 and 4) are collectively referred to as slow-wave sleep (SWS), which is believed to be the most restorative type of sleep and typically occurs during the first one-third of the night. ${ }^{2,8,9}$ In contrast, REM sleep increases as the night progresses and is longest in the last one-third of a sleep episode. ${ }^{2}$ REM and NREM sleep are characterized by numerous, yet different, physiologic changes, including brain activity, heart rate, blood pressure (BP), sympathetic nervous system activity, muscle tone, blood flow to the brain, respiration, airway resistance, renal function, endocrine function, body temperature, and sexual arousal. ${ }^{2}$ For example, during NREM sleep, heart rate, BP, blood flow to the brain, and respiration are decreased compared with wakeful periods. During REM sleep, these processes are increased compared with NREM sleep. Brain activity decreases from wakefulness during NREM sleep; activity levels are similar during REM sleep, except for increases in motor and sensory areas. ${ }^{2}$

A newer sleep classification system developed by the American Academy of Sleep Medicine has only three stages of NREM sleep: lighter sleep (Stages N1 and N2) and deeper sleep (or SWS; Stage N3). ${ }^{10}$ The major changes with the newer classification system are focused on electroencephalogram (EEG) derivations and the merging of Stages 3 and 4 into Stage N3. ${ }^{11}$ In a comparison of the two sleep classifications, only minor differences were noted for total sleep time, sleep efficiency, and REM sleep, but the choice of classification impacted the measurement of wake after sleep onset and the distribution of NREM sleep stages. ${ }^{11}$

The two-process model describes the interplay between the sleep-promoting process (process $\mathrm{S}$ ) and the maintenance of wakefulness system (process C). ${ }^{2}$ The balance between these processes shifts throughout the course of the day, leading to regulation of the sleep-wake cycle. This sleepwake cycle is controlled by daily rhythms of physiology and behavior, called circadian rhythms. ${ }^{2}$ Circadian rhythms also control metabolic activity through physical activity and food consumption, as well as body temperature, heart rate, muscle tone, and hormone secretion. ${ }^{2}$ The sleep process is regulated by neurons in the hypothalamus, which turn off the arousal systems in order to allow sleep to occur. ${ }^{2}$ Insomnia results from the loss of these neurons. Other brain regions are also involved in sleep disruption, including the brain stem and cognitive areas of the forebrain. Over the course of the night, neurons in the pons switch between NREM and REM sleep by sending outputs to the brain stem and spinal cord, 
causing muscle atonia and chaotic autonomic activity; to the forebrain; and to the thalamus via cholinergic pathways. ${ }^{2}$

The circadian rhythms work to synchronize sleep with the external day-night cycle, via the suprachiasmatic nucleus (SCN) that receives direct input from nerve cells in the retina acting as brightness detectors., ${ }^{2,12}$ Light travels from the retina to the $\mathrm{SCN}$, which signals the pineal gland to control the secretion of melatonin. This neurohormone acts to synchronize the circadian rhythms with the environment and the body through melatonin receptors in nearly all tissues. The $\mathrm{SCN}$ also works with a series of clock genes to synchronize the peripheral tissues, giving rise to daily patterns of activity.

\section{Overview of sleep disruption}

Disruption of sleep is widespread. A 2014 survey conducted by the National Sleep Foundation reported that 35\% of American adults rated their sleep quality as "poor" or "only fair". ${ }^{13}$ Trouble falling asleep at least one night per week was reported by $45 \%$ of respondents. ${ }^{13}$ In addition, $53 \%$ of respondents had trouble staying asleep on at least one night of the previous week, and $23 \%$ of respondents had trouble staying asleep on five or more nights. ${ }^{13}$ Snoring was reported by $40 \%$ of respondents, ${ }^{13}$ and $17 \%$ of respondents had been told by a physician that they have a sleep disorder, the majority (68\%) of which was sleep apnea. ${ }^{13}$ Relatively few studies have looked at sleep disruption in children. In a study that included a random sample of Chinese children aged 5-12 years, the overall prevalence of chronic sleep disruption was $9.8 \%$ (boys, $10.0 \%$; girls, $8.9 \%$ ). ${ }^{14}$

Risk factors for sleep disruption are vast and involve a combination of biologic, psychologic, genetic, and social factors (Table 1). ${ }^{2,6,15-39}$ Lifestyle factors include consuming excessive amounts of caffeine ${ }^{15}$ and drinking alcohol. ${ }^{16}$ Performing shift work $^{20}$ or being a college student ${ }^{2}$ is also a risk factor for sleep disruption. Exposure to excessive nighttime light pollution and underexposure to daytime sunlight can lead to disruption of circadian rhythms. ${ }^{19}$ Stressful life circumstances, such as being the parent of a young infant ${ }^{21}$ or serving as a caregiver for a family member with a chronic, life-threatening, or terminal illness, ${ }^{22-25}$ are also contributors to sleep problems. In addition to the stress and worry associated with caregiving, caregivers of patients with complex medication schedules may experience sleep disruption due to the requirement to wake themselves during the night to administer medication. ${ }^{25}$

Sleep disruption is frequently attributable to a sleep disorder, such as obstructive sleep apnea ${ }^{26,27}$ and restless legs syndrome, which is related to altered dopamine and iron metabolism; $>50 \%$ of idiopathic cases of restless leg syndrome have a positive family history. ${ }^{28,29}$ Many major medical condi-
Table I Risk factors contributing to sleep deprivation and disruption

\begin{tabular}{|c|c|}
\hline Category & Risk factors \\
\hline Lifestyle & $\begin{array}{l}\text { - Consuming excessive amounts of caffeine } \\
\text { - Drinking alcohol } \\
\text { - Drug abuse } \\
\text { - Shift work } \\
\text { - Attending university } \\
\text { - Jet lag }\end{array}$ \\
\hline Environmental & $\begin{array}{l}\text { - Excessive noise, such as industrial wind } \\
\text { turbines } \\
\text { - Excessive light }\end{array}$ \\
\hline Psychosocial & $\begin{array}{l}\text { - Anxiety, worry, and rumination } \\
\text { - Parents of young children } \\
\text { - Caregivers to a family member with a chronic, } \\
\text { life-threatening, or terminal illness }\end{array}$ \\
\hline Sleep disorder & $\begin{array}{l}\text { - Insomnia } \\
\text { - Obstructive sleep apnea } \\
\text { - Restless leg syndrome } \\
\text { - Narcolepsy } \\
\text { - Circadian rhythm disorders }\end{array}$ \\
\hline Medical conditions & $\begin{array}{l}\text { - Pain } \\
\text { - Restrictive lung disease } \\
\text { - Chronic kidney disease } \\
\text { - Diabetes } \\
\text { - Neurodegenerative diseases } \\
\text { - Psychiatric disorders } \\
\text { - Use of certain medications }\end{array}$ \\
\hline
\end{tabular}

Note: Data from the following references. .,6, $15-19^{-19}$

tions have been associated with sleep disruption, particularly those that require nighttime medical monitoring (eg, continuous glucose monitoring for individuals with diabetes) ${ }^{38}$ or hospitalization, especially in an intensive or critical care unit. ${ }^{39,40}$

Sleep deprivation studies and studies of insomniacs have identified the primary mechanisms by which sleep disruption is believed to exert its detrimental short- and long-term health effects (Figure 1). ${ }^{41-44}$ During both brief and extended arousals during sleep, increased metabolism is evidenced by increased oxygen consumption and carbon dioxide production..$^{43}$ Levels of catecholamine, norepinephrine, and epinephrine have been correlated with fragmented sleep. ${ }^{44}$ In addition, chronic persistent insomnia is associated with increased secretion of adrenocorticotropic hormone and cortisol, which is present throughout a 24-hour sleep-wake cycle. ${ }^{42}$ These findings suggest that activations of the sympathetic nervous system, the sympathoadrenal system, and the hypothalamic-pituitary-adrenal axis are involved in the health consequences of sleep disruption. ${ }^{41-44}$ In addition, suppression of SWS was associated with decreased insulin sensitivity that did not result in an increase in insulin release; these findings may explain the increased risk of type 2 diabetes mellitus (T2DM) in patients with poor sleep quality. ${ }^{9}$ Other metabolic changes include decreased leptin and increased ghrelin that may contribute to increased appetite. ${ }^{45}$ 


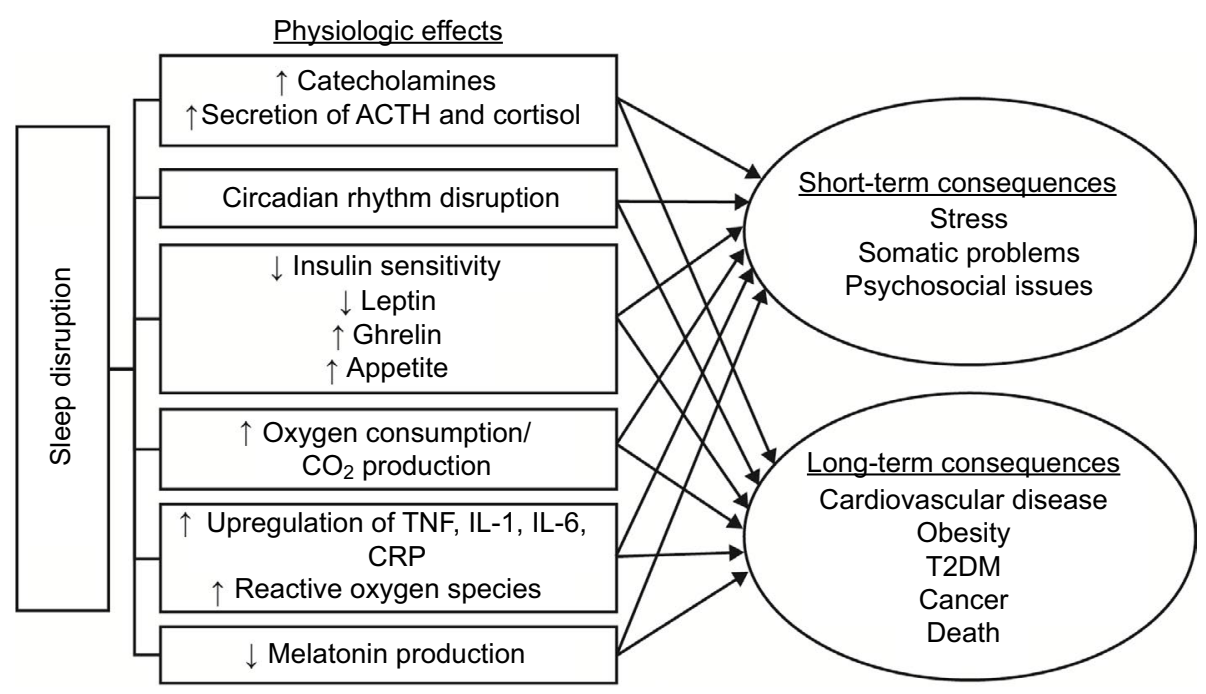

Figure I Proposed mechanisms by which sleep disruption is thought to exert its detrimental short- and long-term effects.

Notes: $\uparrow$, increase; $\downarrow$, decrease. Data from the following references. ${ }^{9} \mid 12,41-45$

Abbreviations: $\mathrm{ACTH}$, adrenocorticotropic hormone; $\mathrm{CO}_{2}$, carbon dioxide; TNF, tumor necrosis factor; IL, interleukin; CRP, C-reactive protein; T2DM, type 2 diabetes mellitus.

Sleep abnormalities affect immune function in a reciprocal manner, leading to changes in proinflammatory cytokines, such as tumor necrosis factor, interleukins 1 and 6, and C-reactive protein. ${ }^{12,46}$ The multitude of systems that react to sleep loss suggest effects beyond the central nervous system and include total body functioning. 5

These wide-ranging effects of sleep disruption are often interrelated and bidirectional. For example, the distress associated with sleep loss can create additional stress to maximize sleep, which, in turn, contributes to worsening (rather than improving) sleep disruption. ${ }^{23}$ The current research suggests that the mechanisms of short- and long-term health consequences are similar but are affected by time. In chronic sleep deprivation, the body's ability to compensate for physiologic changes is diminished, leading to gradually accumulating effects and basal changes. ${ }^{47}$ Insomniacs have been shown to have increased EEG activity, abnormal hormone secretion, increased metabolic activity, and increased sympathetic nervous system activity throughout the day and night. Over time, this heightened and abnormal activity, resulting from the lack of appropriate body rest, can lead to the development of disease and chronic conditions. ${ }^{45}$ Further, insufficient sleep may contribute to alterations in the neuroendocrine stress response system, ultimately leading to stress-related disorders such as mood disorders and depression. ${ }^{47}$

\section{Short-term health consequences of sleep disruption}

As a result of the physiologic changes associated with sleep disruption, numerous health consequences have been reported. Short-term consequences of sleep disruption include increased stress responsivity; somatic problems; reduced quality of life (QoL); emotional distress; mood disorders and other mental health problems; cognition, memory, and performance deficits; and behavior problems in otherwise healthy individuals. Sleep disruption may also diminish the health-related quality of life (HRQoL) of children and adolescents with underlying medical conditions.

\section{Short-term consequences in otherwise healthy individuals}

\section{Increased stress responsivity}

Increased autonomic sympathetic activation is a consequence of fragmented and interrupted sleep ${ }^{47}$ Results of experimental studies suggest that the extent of increased sympathetic activation was related more to the disruption and discontinuity of sleep than to the duration of sleep deprivation or the amount of sleep that was lost. ${ }^{44,48,49}$ Sympathoadrenal activation produces a combination of transient hemodynamic, vasoconstrictive, and prothrombotic processes associated with a stress response. ${ }^{48}$ These effects of sleep disruption on nocturnal regulation of sympathetic activity may offer a connection between sleep disruption and cardiovascular disease (CVD) as well as psychiatric conditions. ${ }^{48}$ By affecting stress hormones, sleep disruption may directly affect functionality, including cognition and mood.

\section{Somatic problems}

A study of adolescents in two Finnish communities found that the 6-month prevalence of weekly sleep problems was 
$27 \%$ and that sleep problems were strongly associated with weekly headache and abdominal pain. ${ }^{50}$ Girls had more symptoms than boys, and an increasing frequency of pain and sleep problems was associated with psychosocial difficulties, such as psychiatric symptoms and substance use. Bidirectional associations between somatic problems and sleep disorders are expected, and these associations may be related to common background factors, such as personality and adverse life events. ${ }^{50}$ During clinical examination, when one symptom is reported, screening for related symptoms should be considered.

\section{Psychosocial issues}

\section{Adults}

Studies have identified a range of psychosocial issues associated with sleep disruption in adults, from emotional distress and mood disorders to cognitive, memory, and performance deficits.

In a qualitative interview-based study by $\mathrm{Neu}$ et al, ${ }^{23}$ mothers of children who were receiving maintenance treatment for acute lymphoblastic leukemia routinely experienced sleep disruption because their children awoke and needed assistance or because of worries related to the child's illness. The mothers reported being irritable, impatient, and less productive than before the illness. In a longitudinal, community-based study of midlife women who had a history of depression and/or anxiety but were not currently ill, sleep disturbance was significantly associated with reduced HRQoL, as measured by the 36-item Short Form Health Survey (SF-36). Odds ratios (ORs) ranged from 2.04 to 2.96, with $P<0.05$ across all HRQoL domains. ${ }^{51}$ A study of 61 maternal caregivers of young children with bronchopulmonary dysplasia showed that $80 \%$ of mothers had clinically disturbed sleep (based on self-report using the Pittsburgh Sleep Quality Index [PSQI]). ${ }^{25}$ This sleep disturbance may be due to the need to administer medication and provide other care during the night, as well as worry about the child's condition. Disrupted sleep was associated with diminished QoL in this study, as assessed using the World Health Organization's Quality of Life Brief. Sleep quality emerged as the only independent variable to significantly predict QoL.

A recent review by Meerlo et al $^{52}$ surveyed the evidence that showed that disrupted sleep is a major causal factor in the development of depression. An experimental study that compared the effects of forced nocturnal awakenings with restricted sleep opportunity and uninterrupted sleep showed that partial sleep loss from sleep continuity disruption was more detrimental to positive mood than partial sleep loss from delaying bedtime. ${ }^{53}$ Adult subjects experiencing forced awakenings had significantly less SWS after the first night of sleep deprivation than other participants. Furthermore, in adults who completed the Personality Assessment Inventory, self-reports of recurring sleep problems were associated with symptoms of depression and anxiety. ${ }^{54}$ The reported frequency of sleep disturbance was closely linked with the severity of the self-reported symptoms. Among primary care physicians, disrupted sleep was associated with high burnout levels. ${ }^{55}$

Sleep disruption alters cognition and performance in many domains, including attention/vigilance, executive function, emotional reactivity, memory formation, decision-making, risk-taking behavior, and judgment. ${ }^{56}$ An experimental study showed that SWS disruption resulted in slower or impaired information processing, impaired sustained attention, less precise motor control, and erroneous implementation of well-practiced actions. ${ }^{57}$ Younger, middle-aged, and older adults were similarly affected by SWS disruption. In another study, poor sleep quality negatively affected the emotional valence of memories. ${ }^{58}$

Across these various studies, the interrelationships between sleep disruption, life events (such as illness of a child), and increased stress responsivity confound the physiologic response. These associations are bidirectional, as anxiety and depression are associated with sleep disruption, and thus make it challenging to separate cause from consequence. ${ }^{47}$ Despite this difficulty, sleep disruption impacts psychosocial functioning in adults and may contribute to psychological conditions that require appropriate intervention.

\section{Adolescents}

Later bedtimes and an inadequate amount of sleep are well-documented changes in sleep patterns associated with adolescence. ${ }^{59}$ A systematic review including 76 studies of the functional consequences of sleep problems in adolescents showed that sleep disruption had a negative effect on psychosocial health, school performance, and risk-taking behaviors, particularly use of nicotine and marijuana. ${ }^{59}$ Studies assessing the relationships between sleep and psychosocial health measures found that sleep disruption was associated with new onset of poor mental health status, ${ }^{60}$ loneliness, ${ }^{61}$ worry, ${ }^{62}$ anxiety, ${ }^{61,63}$ and depression. ${ }^{63}$ In a study of 1,629 adolescents, those with excellent academic performance had earlier bedtimes and longer sleep on weekdays with less severe daytime sleepiness than those with poor grades. ${ }^{64}$ Other studies showed an association between sleep quality and sleep deprivation with poor academic performance. ${ }^{65,66}$ 
Adolescent risk behaviors associated with sleep disruption included cigarette smoking, ${ }^{67,68}$ drinking alcohol, ${ }^{68,69}$ illicit drug use ${ }^{68}$ and aggressive behaviors, including driving while intoxicated, considering suicide, and having unprotected sex. ${ }^{59,62,68}$

Psychosocial outcomes such as depression and mood disturbances, risk-taking behavior, and academic performance appear to be the primary factors affected by sleep disruption in adolescents. Taken together, causal bidirectional relationships are apparent between sleep and psychosocial health as noted earlier for adults. ${ }^{59}$ These findings must be interpreted with caution, however, as many studies of sleep disturbance in adolescents group together the effects of short sleep duration (a common complaint among adolescents) and sleep disruption.

\section{Children}

In a real-world study of 135 healthy children, diminished performance on neurobehavioral functioning measures (particularly those associated with more complex tasks, such as a continuous performance test and a symbol-digit substitution test) were found in children with fragmented sleep. ${ }^{70}$ Parents of these children also rated them as having more behavioral problems than those with continuous sleep. Other reported issues include psychiatric symptoms, ${ }^{71}$ social problems, ${ }^{72}$ externalizing symptoms, ${ }^{71}$ and self-harm behaviors. ${ }^{73}$

\section{Short-term consequences in individuals with underlying medical conditions Reduced QoL}

Of 159 children and adolescents with chronic kidney disease (pre-dialysis, dialysis, and transplant patients), 58.5\% had symptoms of sleep disturbance, as measured by the Epworth Sleepiness Scale. ${ }^{34}$ The presence of a sleep disturbance was most frequent in the dialysis group compared to the other groups, ${ }^{34}$ while sleep disturbance was associated with a significant decrease in the overall total QoL score on the Pediatric Quality of Life Inventory (PedsQL) Version 4.0 Generic Core Scales for pre-dialysis and transplant subjects ( $P=0.002$ and $P=0.001$, respectively). A study of 47 pediatric liver transplant recipients investigated the impact of sleep problems (as assessed by the Pediatric Sleep Questionnaire) on HRQoL, as measured using the PedsQL. ${ }^{74}$ Sleep-related breathing disorders and excessive daytime sleepiness were prevalent, affecting $\sim 23 \%$ and $40 \%$ of children in the study, respectively. ${ }^{74}$ According to the parent proxy and child selfreport, $\sim 40 \%$ of participants had a substandard HRQoL. The physical manifestations of chronic diseases, such as chemical imbalances in dialysis patients, along with medications that may adversely affect sleep, play a role in sleep disruption and require comprehensive management to allow for effective sleep. ${ }^{34,74}$

\section{Long-term health consequences of sleep disruption}

Long-term consequences of sleep disruption in otherwise healthy individuals include hypertension, dyslipidemia, CVD, weight-related issues, metabolic syndrome, and T2DM. Evidence suggests that sleep disruption may increase the risk of certain cancers and death. Sleep disruption may also worsen the symptoms of some gastrointestinal disorders.

\section{Long-term consequences in otherwise healthy individuals}

\section{Cardiovascular}

The increased activity of the sympathetic nervous system that is associated with sleep deprivation has substantial long-term consequences for adults and adolescents. ${ }^{45,47,75-79}$ Adults who experienced sleep disruption had elevated $\mathrm{BP}^{70}$ and an increased risk of developing hypertension. ${ }^{76-78}$ A metaanalysis of data from four prospective cohort studies found that the relative risk of incident hypertension was $1.20(95 \%$ confidence interval [CI], 1.06-1.36) in adults with sleep continuity disturbance, with equal effects in men and women. ${ }^{45}$ In adolescents, higher sleep disturbance scores on the PSQI were associated with higher cholesterol, higher body mass index (BMI), higher systolic BP, and an increased risk of hypertension. ${ }^{79}$ Two large, population-based studies assessed the association between CVD and sleep disruption. ${ }^{76,80}$ In the prospective, population-based Atherosclerosis Risk in Communities (ARIC) Study, incident CVD was observed in patients who experienced sleep continuity disturbance in combination with difficulty falling asleep and nonrestorative sleep (OR, 1.5; 95\% CI, 1.1-2.0). ${ }^{76}$ An association between difficulty maintaining sleep or short sleep duration and incident myocardial infarction was observed in middle-aged women who participated in the MONICA/KORA Augsburg Cohort Study. ${ }^{80}$ Despite differences in study design and populations enrolled, these studies extend the literature to suggest that the effects of sleep disruption on sympathetic activity, glucose metabolism, and possibly inflammation may lead to adverse cardiovascular effects. ${ }^{80}$

\section{Metabolic}

A recent review by Cedernaes et al $^{81}$ described a variety of molecular and behavioral factors that may lead to an asso- 
ciation between sleep disruption and metabolic disorders, including obesity and T2DM. Sleep loss appears to affect energy metabolism primarily by impairing insulin sensitivity and increasing food intake. ${ }^{81}$ Disrupted sleep has been associated with weight gain and other weight-related issues in both adults ${ }^{82,83}$ and adolescents. ${ }^{79}$ A 5-year ancillary study nested within the Coronary Artery Risk Development in Young Adults (CARDIA) study showed that sleep fragmentation was strongly associated with increases in BMI. ${ }^{82} \mathrm{~A}$ common cause of sleep disruption is shift work, which has been implicated in high BP and increased stress. ${ }^{20}$ A 14-year longitudinal study in male Japanese workers showed that alternating shift work increased the rate of everyday drinking, smoking, and absence of habitual exercise and also heightened the risk of increasing BMI. ${ }^{83}$ In adolescents, sleep disruption was associated with a high BMI $z$-score, being overweight, and having a high waist circumference percentile. ${ }^{79}$

The results of experimental studies in healthy volunteers suggest that, independent of sleep duration, sleep fragmentation can alter glucose homeostasis. ${ }^{9}$ In an experimental study in healthy young adults, sleep disruption (characterized by three nights of SWS suppression) resulted in decreased insulin sensitivity, which was similar to that reported for populations at high risk of T2DM, and reduced glucose tolerance. ${ }^{9}$ Other experimental studies showed that sleep fragmentation resulted in reduced insulin sensitivity, reduced glucose effectiveness (defined as the ability of glucose to mobilize itself independent of an insulin response), and increased cortisol levels. ${ }^{84,85}$ Large longitudinal studies have shown that sleep disruption is associated with an increased risk of developing T2DM. ${ }^{78,86-89}$ A meta-analysis of four of these studies ${ }^{86-89}$ found that the overall relative risk of developing T2DM was 1.84 (95\% CI, 1.39-2.43; $P<0.0001)$ in adults who experienced difficulty maintaining sleep..$^{90}$

The coexistence of obesity, elevated BP and glucose levels, and low levels of high-density lipoprotein cholesterol defines the metabolic syndrome. ${ }^{91}$ An observational, crosssectional study compared global scores on the PSQI with concurrently collected measures of metabolic syndrome components. ${ }^{91}$ Poor global sleep-quality scores on the PSQI were related significantly to the presence of metabolic syndrome, and the PSQI global sleep-quality score was significantly related to waist circumference, BMI, percentage of body fat, serum levels of insulin and glucose, and estimated insulin resistance.

The accumulating evidence points to the importance of regular sleep for normal metabolic functioning and prevention of the metabolic syndrome. ${ }^{81}$ The metabolic effects of sleep disruption appear to manifest in both the brain and peripheral organs. The effects of sleep disruption on appetite, glucose metabolism, and diabetes risk are critical to understanding the epidemic of obesity and metabolic disease. It has even been suggested that sleep may be an appropriate therapeutic target for treatment and prevention of obesity and diabetes. ${ }^{81}$

\section{Cancer}

Disruption of circadian rhythm and sleep deprivation have been shown to accelerate tumor formation ${ }^{12}$ and may increase the risk of cancer. ${ }^{12,92}$ Exposure to light at night decreases production of melatonin, which may lead to increased production of reproductive hormones. ${ }^{93}$ Melatonin has other important properties, including DNA repair, inhibition of tumor growth, and acting as a potent free-radical scavenger. ${ }^{92,94}$ A study in mice subjected to suprachiasmatic nuclei destruction showed that disruption of circadian coordination accelerated malignant growth, which suggests that the host circadian clock controls tumor progression ${ }^{95}$ and provides a potential mechanistic reason for this association.

With regard to clinical data, night shift work has been associated with an increased risk of cancer. In the Nurses' Health Study, 602 incident cases of colorectal cancer were documented among 78,586 women who were followed over 10 years. ${ }^{93}$ Compared with women who never worked rotating night shifts, women who worked 1-14 years or $\geq 15$ years on rotating night shifts had multivariate relative risks of colorectal cancer of 1.00 (95\% CI, 0.84-1.19) and 1.35 (95\% CI, 1.03-1.77), respectively $\left(P_{\text {trend }}=0.04\right)$. These data suggest that extended night shift work may increase the risk of colorectal cancer. Moreover, men who suffered from severe problems of falling and staying asleep were about twice as likely to develop prostate cancer as those without insomnia. ${ }^{92}$

A recent large nested case-control study from Taiwan determined an increased risk of cancer among patients with sleep disorders compared with those without sleep disorders. ${ }^{96}$ In this study, sleep disorders were separated into three categories: insomnia, parasomnia, and obstructive sleep apnea, all of which can contribute to sleep disruption. The risk of breast cancer was increased for patients with each of these types of disorder (adjusted hazard ratio 1.73 [95\% CI, 1.57-1.90] for insomnia, 2.76 [95\% CI, 1.53-5.00] for parasomnia, 2.10 [95\% CI, 1.16-3.80] for obstructive sleep apnea). There was also a higher risk of nasal cancer and prostate cancer in patients with obstructive sleep apnea compared with those without sleep disruptions. 
The mechanisms responsible for carcinogenesis in sleepdisrupted individuals are not clear, and much of the work is focused on nighttime light exposure and decreased melatonin levels. ${ }^{92,93}$ Additional research is required to determine the effect and etiology of sleep disruption on cancer risk.

\section{Death}

In the GAZEL cohort study that assessed sleep disturbances using the 5-item sleep dimension from the Nottingham Health Profile, sleep disturbance was associated with a higher all-cause risk of mortality in men $(P=0.005)$, but not in women $(P=0.33)$. In particular, men who reported sleep disruption on the Nottingham Health Profile ("I sleep badly at night") had a higher all-cause mortality risk compared with those who did not report sleep disruption (hazard ratio, 1.69; 95\% CI, 1.25-2.31). ${ }^{78}$ In a study in which the family and friends of adolescent suicide completers reported sleep disturbances for the deceased, history of sleep disturbances, including middle insomnia, was significantly associated with suicide compared with matched community controls. ${ }^{97}$ The effect remained significant when controlling for current affective disorders and severity of depressive symptoms.

The high correlation between sleep disturbances, depression, and suicidal ideation may play a role in identifying an increased risk of mortality in these studies. Other studies have linked sleep disorders to mortality through an increase in cardiovascular deaths, which have also been related to sleep disruption. Additional studies are needed in larger cohorts and controlling for confounding factors. Importantly, hypertension and diabetes may not explain death in younger individuals with sleep disruption, but the association of sleep disruption with these factors is a risk factor for mortality in later life. ${ }^{78}$

\section{Long-term consequences in individuals with underlying medical conditions}

The interdependent relationship between sleep and the immune system may be a factor in the effect of sleep abnormalities on common gastrointestinal disorders. Sleep disruption may worsen symptoms of inflammatory bowel disease, irritable bowel syndrome, and gastroesophageal reflux disease. ${ }^{12}$ Conversely, these same gastrointestinal disorders can also contribute to sleep disruption. As seen with many other consequences of sleep disruption, the bidirectional interplay between sleep disruption and gastrointestinal disorders provides the opportunity for clinicians to treat both conditions for improved patient outcomes.

\section{Conclusion}

Disrupted sleep is a pervasive problem, with numerous contributing factors from lifestyle and environmental factors to psychosocial issues and iatrogenic effects. Sleep is vital to most major physiologic processes, and, as such, sleep disruption has vast potential for adverse short- and long-term health consequences in otherwise healthy individuals as well as those with underlying medical conditions. In healthy individuals, short-term consequences include a heightened stress response; pain; depression; anxiety; and cognition, memory, and performance deficits. In adolescents and children, disrupted sleep can lead to poor school performance and behavior problems. Reduced QoL may be a short-term consequence of sleep disruption in otherwise healthy individuals and those with an underlying medical condition. Long-term consequences for otherwise healthy individuals include hypertension, dyslipidemia, CVD, weight gain, metabolic syndrome, and T2DM. There is also evidence that sleep disruption may increase the risk of certain cancers and death in males and suicidal adolescents. Long-term sleep disruption may also worsen the symptoms of a variety of gastrointestinal disorders.

Ultimately, it has been suggested that the physiologic consequences of disrupted sleep may be just as damaging as those of short sleep duration. ${ }^{5}$ Given the detrimental impact of disrupted sleep, it is important for health care professionals to effectively treat symptoms of underlying medical conditions to optimize sleep continuity. In addition, when possible, health care providers should consider prescribing interventions that minimize disruptions to sleep continuity, ${ }^{25}$ such as medications with a long dosing interval.

\section{Acknowledgment}

Medical writing assistance for this manuscript was provided by Katie Gersh, PhD, of MedErgy and was funded by Horizon Pharma.

\section{Disclosure}

All authors are employees of Horizon Pharma, which funded medical writing assistance for this manuscript. The authors report no other conflicts of interest in this work.

\section{References}

1. Watson NF, Badr MS, Belenky G, et al. Joint Consensus Statement of the American Academy of Sleep Medicine and Sleep Research Society on the recommended amount of sleep for a healthy adult: methodology and discussion. Sleep. 2015;38(8):1161-1183.

2. Institute of Medicine, Committee on Sleep Medicine and Research, Board on Health Sciences Policy. Sleep Disorders and Sleep Deprivation: An Unmet Public Health Problem. Washington, DC: National Academies Press; 2006. 
3. Watson NF, Badr MS, Belenky G, et al. Recommended amount of sleep for a healthy adult: a joint consensus statement of the American Academy of Sleep Medicine and Sleep Research Society. Sleep. 2015; 38(6):843-844.

4. Olesen J, Gustavsson A, Svensson M, et al; CDBE2010 Study Group; European Brain Council. The economic cost of brain disorders in Europe. Eur J Neurol. 2012;19(1):155-162.

5. Van Someren EJ, Cirelli C, Dijk DJ, Van CE, Schwartz S, Chee MW. Disrupted sleep: from molecules to cognition. J Neurosci. 2015 35(41):13889-13895.

6. Abbott SM, Videnovic A. Chronic sleep disturbance and neural injury: links to neurodegenerative disease. Nat Sci Sleep. 2016;8:55-61.

7. Kim TW, Jeong JH, Hong SC. The impact of sleep and circadian disturbance on hormones and metabolism. Int J Endocrinol. 2015; 2015:591729.

8. Rechtschaffen A, Kales A. A Manual of Standardized Terminology Techniques and Scoring System for Sleep Stages of Human Subjects. Bethesda, MD: National Institutes of Health, National Institute of Neurological Diseases and Blindness, Neurological Information Network; 1968.

9. Tasali E, Leproult R, Ehrmann DA, Van CE. Slow-wave sleep and the risk of type 2 diabetes in humans. Proc Natl Acad Sci U S A. 2008; 105(3):1044-1049.

10. Iber C, Ancoli-Israel S, Chesson A, Quan SF. The AASM Manual for the Scoring of Sleep and Associated Events: Rules, Terminology, and Technical Specifications. Westchester, IL: American Academy of Sleep Medicine; 2007.

11. Moser D, Anderer P, Gruber G, et al. Sleep classification according to AASM and Rechtschaffen \& Kales: effects on sleep scoring parameters. Sleep. 2009;32(2):139-149.

12. Ali T, Choe J, Awab A, Wagener TL, Orr WC. Sleep, immunity and inflammation in gastrointestinal disorders. World $J$ Gastroenterol. 2013;19(48):9231-9239.

13. National Sleep Foundation. 2014 Sleep Health Index. Arlington, VA: National Sleep Foundation; 2014.

14. Li L, Ren J, Shi L, et al. Frequent nocturnal awakenings in children: prevalence, risk factors, and associations with subjective sleep perception and daytime sleepiness. BMC Psychiatry. 2014;14:204.

15. Clark I, Landolt HP. Coffee, caffeine, and sleep: a systematic review of epidemiological studies and randomized controlled trials. Sleep Med Rev. 2017;31:70-78.

16. Thakkar MM, Sharma R, Sahota P. Alcohol disrupts sleep homeostasis. Alcohol. 2015;49(4):299-310.

17. Lipinska G, Timol R, Thomas KG. The implications of sleep disruption for cognitive and affective processing in methamphetamine abuse. Med Hypotheses. 2015;85(6):914-921.

18. Nissenbaum MA, Aramini JJ, Hanning CD. Effects of industrial wind turbine noise on sleep and health. Noise Health. 2012; 14(60):237-243.

19. Smolensky MH, Sackett-Lundeen LL, Portaluppi F. Nocturnal light pollution and underexposure to daytime sunlight: complementary mechanisms of circadian disruption and related diseases. Chronobiol Int. 2015;32(8):1029-1048.

20. Boivin DB, Boudreau P. Impacts of shift work on sleep and circadian rhythms. Pathol Biol (Paris). 2014;62(5):292-301.

21. Malish S, Arastu F, O’Brien LM. A preliminary study of new parents, sleep disruption, and driving: a population at risk? Matern Child Health J. 2016;20(2):290-297.

22. Celik G, Annagur BB, Yilmaz M, Demir T, Kara F. Are sleep and life quality of family caregivers affected as much as those of hemodialysis patients? Gen Hosp Psychiatry. 2012;34(5):518-524.

23. Neu M, Matthews E, King NA. Exploring sleep-wake experiences of mothers during maintenance therapy for their child's acute lymphoblastic leukemia. J Pediatr Nurs. 2014;29(5):410-421.

24. Lerdal A, Gay CL, Saghaug E, et al. Sleep in family caregivers of patients admitted to hospice: a pilot study. Palliat Support Care. 2014;12(6):439-444.
25. Feeley CA, Turner-Henson A, Christian BJ, et al. Sleep quality, stress, caregiver burden, and quality of life in maternal caregivers of young children with bronchopulmonary dysplasia. J Pediatr Nurs. 2014;29(1):29-38.

26. Ryu HS, Lee SA, Lee GH, Chung YS, Kim WS. Subjective apnea symptoms are associated with daytime sleepiness in patients with moderate and severe obstructive sleep apnea: a retrospective study. Clin Otolaryngol. 2016;41(4):395-401.

27. Younes M, Hanly PJ. Immediate postarousal sleep dynamics: an important determinant of sleep stability in obstructive sleep apnea. J Appl Physiol (1985). 2016;120(7):801-808.

28. Ferri R, Rundo F, Zucconi M, et al. An evidence-based analysis of the association between periodic leg movements during sleep and arousals in restless legs syndrome. Sleep. 2015;38(6):919-924.

29. Fehnel S, Zografos L, Curtice T, Shah H, McLeod L. The burden of restless legs syndrome: an assessment of work productivity, sleep, psychological distress, and health status among diagnosed and undiagnosed individuals in an internet-based panel. Patient. 2008;1(3):201-210.

30. Boakye PA, Olechowski C, Rashiq S, et al. A critical review of neurobiological factors involved in the interactions between chronic pain, depression, and sleep disruption. Clin J Pain. 2016;32(4):327-336.

31. Gupta MA, Gupta AK. Sleep-wake disorders and dermatology. Clin Dermatol. 2013;31(1):118-126.

32. Won $\mathrm{CH}$, Kryger M. Sleep in patients with restrictive lung disease. Clin Chest Med. 2014;35(3):505-512.

33. Barmar B, Dang Q, Isquith D, Buysse D, Unruh M. Comparison of sleep/wake behavior in CKD stages 4 to 5 and hemodialysis populations using wrist actigraphy. Am J Kidney Dis. 2009;53(4):665-672.

34. Davis ID, Greenbaum LA, Gipson D, et al. Prevalence of sleep disturbances in children and adolescents with chronic kidney disease. Pediatr Nephrol. 2012;27(3):451-459.

35. Brod M, Wolden M, Christensen T, Bushnell DM. A nine country study of the burden of non-severe nocturnal hypoglycaemic events on diabetes management and daily function. Diabetes Obes Metab. 2013; 15(6):546-557.

36. Kamath J, Virdi S, Winokur A. Sleep disturbances in schizophrenia. Psychiatr Clin North Am. 2015;38(4):777-792.

37. Boland EM, Stange JP, Molz AA, et al. Associations between sleep disturbance, cognitive functioning and work disability in bipolar disorder. Psychiatry Res. 2015;230(2):567-574.

38. Bailey TS, Grunberger G, Bode BW, et al. American Association of Clinical Endocrinologists and American College of Endocrinology 2016 outpatient glucose monitoring consensus statement. Endocr Pract. 2016;22(2):231-261.

39. Pulak LM, Jensen L. Sleep in the intensive care unit: a review. J Intensive Care Med. 2016;31(1):14-23.

40. Elliott R, Rai T, McKinley S. Factors affecting sleep in the critically ill: an observational study. J Crit Care. 2014;29(5):859-863.

41. Vgontzas AN, Tsigos C, Bixler EO, et al. Chronic insomnia and activity of the stress system: a preliminary study. J Psychosom Res. 1998;45(1):21-31.

42. Vgontzas AN, Bixler EO, Lin HM, et al. Chronic insomnia is associated with nyctohemeral activation of the hypothalamic-pituitary-adrenal axis: clinical implications. J Clin Endocrinol Metab. 2001;86(8):3787-3794.

43. Bonnet MH, Berry RB, Arand DL. Metabolism during normal, fragmented, and recovery sleep. J Appl Physiol (1985). 1991;71(3): 1112-1118.

44. Tiemeier H, Pelzer E, Jonck L, Moller HJ, Rao ML. Plasma catecholamines and selective slow wave sleep deprivation. Neuropsychobiology. 2002;45(2):81-86.

45. Meng L, Zheng Y, Hui R. The relationship of sleep duration and insomnia to risk of hypertension incidence: a meta-analysis of prospective cohort studies. Hypertens Res. 2013;36(11):985-995.

46. Hurtado-Alvarado G, Dominguez-Salazar E, Pavon L, VelazquezMoctezuma J, Gomez-Gonzalez B. Blood-brain barrier disruption induced by chronic sleep loss: low-grade inflammation may be the link. J Immunol Res. 2016;2016:4576012. 
47. Meerlo P, Sgoifo A, Suchecki D. Restricted and disrupted sleep: effects on autonomic function, neuroendocrine stress systems and stress responsivity. Sleep Med Rev. 2008;12(3):197-210.

48. Irwin M, Thompson J, Miller C, Gillin JC, Ziegler M. Effects of sleep and sleep deprivation on catecholamine and interleukin-2 levels in humans: clinical implications. J Clin Endocrinol Metab. 1999; 84(6):1979-1985.

49. Ekstedt M, Akerstedt T, Soderstrom M. Microarousals during sleep are associated with increased levels of lipids, cortisol, and blood pressure. Psychosom Med. 2004;66(6):925-931.

50. Luntamo T, Sourander A, Rihko M, et al. Psychosocial determinants of headache, abdominal pain, and sleep problems in a community sample of Finnish adolescents. Eur Child Adolesc Psychiatry. 2012; 21(6):301-313.

51. Joffe H, Chang Y, Dhaliwal S, et al. Lifetime history of depression and anxiety disorders as a predictor of quality of life in midlife women in the absence of current illness episodes. Arch Gen Psychiatry. 2012;69(5):484-492.

52. Meerlo P, Havekes R, Steiger A. Chronically restricted or disrupted sleep as a causal factor in the development of depression. Curr Top Behav Neurosci. 2015;25:459-481.

53. Finan PH, Quartana PJ, Smith MT. The effects of sleep continuity disruption on positive mood and sleep architecture in healthy adults. Sleep. 2015;38(11):1735-1742.

54. Tkachenko O, Olson EA, Weber M, Preer LA, Gogel H, Killgore WD. Sleep difficulties are associated with increased symptoms of psychopathology. Exp Brain Res. 2014;232(5):1567-1574.

55. Vela-Bueno A, Moreno-Jimenez B, Rodriguez-Munoz A, et al. Insomnia and sleep quality among primary care physicians with low and high burnout levels. J Psychosom Res. 2008;64(4):435-442.

56. McCoy JG, Strecker RE. The cognitive cost of sleep lost. Neurobiol Learn Mem. 2011;96(4):564-582.

57. Groeger JA, Stanley N, Deacon S, Dijk DJ. Dissociating effects of global SWS disruption and healthy aging on waking performance and daytime sleepiness. Sleep. 2014;37(6):1127-1142.

58. Tempesta D, De GL, Natale V, Ferrara M. Emotional memory processing is influenced by sleep quality. Sleep Med. 2015;16(7):862-870.

59. Shochat T, Cohen-Zion M, Tzischinsky O. Functional consequences of inadequate sleep in adolescents: a systematic review. Sleep Med Rev. 2014;18(1):75-87.

60. Kaneita Y, Yokoyama E, Harano S, et al. Associations between sleep disturbance and mental health status: a longitudinal study of Japanese junior high school students. Sleep Med. 2009;10(7):780-786.

61. Xu Z, Su H, Zou Y, Chen J, Wu J, Chang W. Sleep quality of Chinese adolescents: distribution and its associated factors. $J$ Paediatr Child Health. 2012;48(2):138-145.

62. Umlauf MG, Bolland JM, Lian BE. Sleep disturbance and risk behaviors among inner-city African-American adolescents. J Urban Health. 2011;88(6):1130-1142.

63. Johnson EO, Roth T, Schultz L, Breslau N. Epidemiology of DSM-IV insomnia in adolescence: lifetime prevalence, chronicity, and an emergent gender difference. Pediatrics. 2006;117(2):e247-e256.

64. Chung KF, Cheung MM. Sleep-wake patterns and sleep disturbance among Hong Kong Chinese adolescents. Sleep. 2008;31(2):185-194.

65. Dewald JF, Meijer AM, Oort FJ, Kerkhof GA, Bogels SM. The influence of sleep quality, sleep duration and sleepiness on school performance in children and adolescents: a meta-analytic review. Sleep Med Rev. 2010;14(3):179-189.

66. Mak KK, Lee SL, Ho SY, Lo WS, Lam TH. Sleep and academic performance in Hong Kong adolescents. $J$ Sch Health. 2012;82(11): $522-527$.

67. Mak KK, Ho SY, Thomas GN, et al. Smoking and sleep disorders in Chinese adolescents. Sleep Med. 2010;11(3):268-273.

68. Perez A, Roberts RE, Sanderson M, Reininger B, Aguirre-Flores MI. Disturbed sleep among adolescents living in 2 communities on the Texas-Mexico border, 2000-2003. Prev Chronic Dis. 2010;7(2):A40.
69. Morioka H, Itani O, Kaneita Y, et al. Associations between sleep disturbance and alcohol drinking: a large-scale epidemiological study of adolescents in Japan. Alcohol. 2013;47(8):619-628.

70. Sadeh A, Gruber R, Raviv A. Sleep, neurobehavioral functioning, and behavior problems in school-age children. Child Dev. 2002;73(2): 405-417.

71. Paavonen EJ, Porkka-Heiskanen T, Lahikainen AR. Sleep quality, duration and behavioral symptoms among 5-6-year-old children. Eur Child Adolesc Psychiatry. 2009;18(12):747-754.

72. Velten-Schurian K, Hautzinger M, Poets CF, Schlarb AA. Association between sleep patterns and daytime functioning in children with insomnia: the contribution of parent-reported frequency of night waking and wake time after sleep onset. Sleep Med. 2010;11(3):281-288.

73. Singareddy R, Krishnamurthy VB, Vgontzas AN, et al. Subjective and objective sleep and self-harm behaviors in young children: a general population study. Psychiatry Res. 2013;209(3):549-553.

74. Fredericks EM, Dore-Stites D, Calderon SY, et al. Relationship between sleep problems and health-related quality of life among pediatric liver transplant recipients. Liver Transpl. 2012;18(6):707-715.

75. Knutson KL, Van CE, Rathouz PJ, et al. Association between sleep and blood pressure in midlife: the CARDIA sleep study. Arch Intern Med. 2009;169(11):1055-1061

76. Phillips B, Mannino DM. Do insomnia complaints cause hypertension or cardiovascular disease? J Clin Sleep Med. 2007;3(5):489-494.

77. Suka M, Yoshida K, Sugimori H. Persistent insomnia is a predictor of hypertension in Japanese male workers. J Occup Health. 2003; 45(6):344-350

78. Rod $\mathrm{NH}$, Vahtera J, Westerlund $\mathrm{H}$, et al. Sleep disturbances and cause-specific mortality: results from the GAZEL cohort study. Am J Epidemiol. 2011;173(3):300-309.

79. Narang I, Manlhiot $C$, vies-Shaw J, et al. Sleep disturbance and cardiovascular risk in adolescents. CMAJ. 2012;184(17):E913-E920.

80. Meisinger C, Heier M, Lowel H, Schneider A, Doring A. Sleep duration and sleep complaints and risk of myocardial infarction in middle-aged men and women from the general population: the MONICA/KORA Augsburg cohort study. Sleep. 2007;30(9):1121-1127.

81. Cedernaes J, Schioth HB, Benedict C. Determinants of shortened, disrupted, and mistimed sleep and associated metabolic health consequences in healthy humans. Diabetes. 2015;64(4):1073-1080.

82. Lauderdale DS, Knutson KL, Rathouz PJ, Yan LL, Hulley SB, Liu $\mathrm{K}$. Cross-sectional and longitudinal associations between objectively measured sleep duration and body mass index: the CARDIA Sleep Study. Am J Epidemiol. 2009;170(7):805-813.

83. Suwazono Y, Dochi M, Sakata K, et al. A longitudinal study on the effect of shift work on weight gain in male Japanese workers. Obesity (Silver Spring). 2008;16(8):1887-1893.

84. Stamatakis KA, Punjabi NM. Effects of sleep fragmentation on glucose metabolism in normal subjects. Chest. 2010;137(1):95-101.

85. Herzog N, Jauch-Chara K, Hyzy F, et al. Selective slow wave sleep but not rapid eye movement sleep suppression impairs morning glucose tolerance in healthy men. Psychoneuroendocrinology. 2013; 38(10):2075-2082.

86. Hayashino Y, Fukuhara S, Suzukamo Y, et al; HIPOP-OHP Research Group. Relation between sleep quality and quantity, quality of life, and risk of developing diabetes in healthy workers in Japan: the High-risk and Population Strategy for Occupational Health Promotion (HIPOPOHP) Study. BMC Public Health. 2007;7:129.

87. Kawakami N, Takatsuka N, Shimizu H. Sleep disturbance and onset of type 2 diabetes. Diabetes Care. 2004;27(1):282-283.

88. Mallon L, Broman JE, Hetta J. High incidence of diabetes in men with sleep complaints or short sleep duration: a 12-year follow-up study of a middle-aged population. Diabetes Care. 2005;28(11):2762-2767.

89. Meisinger C, Heier M, Loewel H. MONICA/KORA Augsburg Cohort Study. Sleep disturbance as a predictor of type 2 diabetes mellitus in men and women from the general population. Diabetologia. 2005;48:235-241. 
90. Cappuccio FP, D’Elia L, Strazzullo P, Miller MA. Quantity and quality of sleep and incidence of type 2 diabetes: a systematic review and meta-analysis. Diabetes Care. 2010;33(2):414-420.

91. Jennings JR, Muldoon MF, Hall M, Buysse DJ, Manuck SB. Selfreported sleep quality is associated with the metabolic syndrome. Sleep. 2007;30(2):219-223.

92. Sigurdardottir LG, Valdimarsdottir UA, Mucci LA, et al. Sleep disruption among older men and risk of prostate cancer. Cancer Epidemiol Biomarkers Prev. 2013;22(5):872-879.

93. Schernhammer ES, Laden F, Speizer FE, et al. Night-shift work and risk of colorectal cancer in the nurses' health study. J Natl Cancer Inst. 2003;95(11):825-828.
94. Thompson CL, Larkin EK, Patel S, Berger NA, Redline S, Li L. Short duration of sleep increases risk of colorectal adenoma. Cancer. 2011;117(4):841-847.

95. Filipski E, King VM, Li X, et al. Disruption of circadian coordination accelerates malignant growth in mice. Pathol Biol (Paris). 2003; 51(4):216-219.

96. Fang HF, Miao NF, Chen CD, Sithole T, Chung MH. Risk of cancer in patients with insomnia, parasomnia, and obstructive sleep apnea: a Nationwide Nested Case-Control Study. J Cancer. 2015;6(11): 1140-1147.

97. Goldstein TR, Bridge JA, Brent DA. Sleep disturbance preceding completed suicide in adolescents. J Consult Clin Psychol. 2008;76(1):84-91.

\section{Publish your work in this journal}

Nature and Science of Sleep is an international, peer-reviewed, open access journal covering all aspects of sleep science and sleep medicine, including the neurophysiology and functions of sleep, the genetics of sleep, sleep and society, biological rhythms, dreaming, sleep disorders and therapy, and strategies to optimize healthy sleep. The manuscript

\section{Dovepress}

management system is completely online and includes a very quick and fair peer-review system, which is all easy to use. Visit http://www. dovepress.com/testimonials.php to read real quotes from published authors. 